\title{
Sucessos terapêuticos obtidos através dos serviços farmacêuticos destinados ao paciente, família e comunidade
}

Therapeutic successes obtained through pharmaceutical services aimed at the patient, family and community

Éxitos terapêuticos obtenidos a través de servicios farmacêuticos dirigidos al paciente, família y la comunidad

\author{
Renato Bruno Cavalcante de Melo \\ ORCID: https://orcid.org/0000-0002-6159-3802 \\ Universidade Federal do Pará, Brasil \\ E-mail: renatobcdemelo@gmail.com \\ Camila Martins Oliveira \\ ORCID: https://orcid.org/0000-0002-6425-2384 \\ Universidade Federal do Pará, Brasil \\ E-mail: cmofarma@gmail.com \\ Clarisse Andrade Sales \\ ORCID: https://orcid.org/0000-0001-6641-4618 \\ Universidade Federal do Pará, Brasil \\ E-mail: clarisseasales@gmail.com \\ Maria Pantoja Moreira de Sena \\ ORCID: https://orcid.org/0000-0001-6641-4618 \\ Universidade Federal do Pará, Brasil \\ E-mail: mariapantojamoreira@ hotmail.com \\ Marcos Felipe Rodrigues de Souza \\ ORCID: https://orcid.org/0000-0002-6246-7525 \\ Universidade Federal do Pará, Brasil \\ E-mail: marcosfrodrigues.br@gmail.com \\ Crystyanne de Sousa Freitas \\ ORCID: https://orcid.org/0000-0003-4279-1587 \\ Universidade Federal do Pará, Brasil \\ E-mail: crystyannefreitas@icloud.com \\ Amanda Gabryelle Nunes Cardoso Mello \\ ORCID: https://orcid.org/0000-0001-7661-1615 \\ Universidade Federal do Pará, Brasil \\ E-mail: agncmello@gmail.com \\ Luann Wendel Pereira de Sena \\ ORCID: https://orcid.org/0000-0001-9363-5766 \\ Universidade Federal do Pará, Brasil \\ E-mail: luannsena@gmail.com
}

\begin{abstract}
Resumo
Objetivo: elaborar uma revisão integrativa acerca dos principais serviços farmacêuticos conferido ao paciente, família e comunidade no Brasil. Métodos: refere-se à verificação bibliográfica e consulta em banco de dados a respeito dos serviços farmacêuticos. Resultados: detectou-se que inúmeras categorias de serviços concebidas no contexto da atenção à saúde, encontram-se expostos e apreciados positivamente no desenvolvimento da qualidade do processo do uso de medicamentos e nas consequências terapêuticos para o usuário. Os serviços providos por farmacêuticos, como o acompanhamento farmacoterapêutico, conciliação medicamentosa, rastreamento em saúde, manejo de problemas de saúde autolimitados e outros, contribuem para o auxílio do cuidado em saúde, sendo estes, essenciais para o uso racional de medicamentos e ao cuidado/segurança atribuídos aos pacientes Conclusão: a inserção dos serviços farmacêuticos exibe um maior reconhecimento das competências do profissional no sistema de atenção à saúde, promovendo o enaltecimento do impacto de sua atuação na melhoria da farmacoterapia, no aprimoramento dos resultados em saúde, na prevenção de doenças e na ascensão de saúde da população brasileira.
\end{abstract}

Palavras-chave: Serviços farmacêuticos; Farmácia clínica; Farmacêutico.

\section{Abstract}

Objective: to prepare an integrative review pharmaceutical services provided to the patient, family and community in Brazil. Methods: refers to bibliographic verification and database consultation about pharmaceutical services. Results: 
it was detected that numerous categories of services conceived in the context are exposed and positively appreciated in the use process and the therapeutic consequences for the user. The services provided by pharmacists, such as pharmacotherapeutic monitoring, medication reconciliation, health screening, management of self-limited health problems and others, which are essential for the rational use of medicines and care / safety attributed to patients Conclusion: shows greater recognition of the professional's skills in the health care system, promoting the enhancement of the impact of their performance on improving pharmacotherapy, improving health outcomes, preventing diseases and in the health rise of the population.

Keywords: Pharmaceutical services; Clinical pharmacy; Pharmacist.

\section{Resumen}

Objetivo: preparar una revisión integradora de los servicios farmacéuticos prestados al paciente, la familia y la comunidad en Brasil. Métodos: se refiere a la verificación bibliográfica y consulta de bases de datos sobre servicios farmacéuticos. Resultados: se detectó que numerosas categorías de servicios concebidos en el contexto se exponen y valoran positivamente en el proceso de uso y las consecuencias terapéuticas para el usuario. Los servicios que prestan los farmacéuticos, como el seguimiento farmacoterapéutico, la conciliación de medicación, el cribado de salud, el manejo de problemas de salud autolimitados y otros, que son fundamentales para el uso racional de los medicamentos y la atención / seguridad atribuida a los pacientes Conclusión: muestra un mayor reconocimiento de la las habilidades de los profesionales en el sistema de atención de la salud, promoviendo la mejora del impacto de su desempeño en la mejora de la farmacoterapia, la mejora de los resultados de salud, la prevención de enfermedades y el aumento de la salud de la población.

Palabras clave: Servicios farmacéuticos; Farmacia clínica; Farmacéutico.

\section{Introdução}

O planejamento e a direção do trabalho no âmbito da saúde são definidos pela necessidade de contribuição entre os profissionais da equipe multidisciplinar, possibilitando o paciente, um apoio abrangente das suas carências de vida, principalmente daquelas pertencentes à saúde (Fernandes et al., 2019). Neste contexto, é importante que os componentes da equipe identifiquem o método de trabalho dos seus semelhantes, a fim de otimizar os resultados (Melo et al., 2021).

Ao observar o exercício do farmacêutico na atenção à saúde, nota-se sua atividade tanto nos sistemas de apoio, quanto na prevenção direta ao paciente, família e comunidade (Araújo et al., 2017). As primeiras se associam à fabricação de medicamentos e de outros itens, tal como, à sua gestão logística, objetivando a introdução do paciente a mecanismos terapêuticos e propedêuticos. O suporte diagnóstico atribuído pelas análises clínicas, também é visto como atividade de apoio. A atividade-fim e/ou de prevenção direta, é aquela que descreve a finalidade principal da instituição. No caso dos sistemas de saúde, as atividades-fim pertencem àquelas relativas ao apoio direto ao paciente, família e comunidade (Souza et al., 2018).

A atenção à saúde desempenhada pelo farmacêutico se concretiza para o paciente e/ou sociedade no fornecimento de serviços farmacêuticos (SF) (Maioli et al., 2018). Os serviços como o acompanhamento farmacoterapêutico (AFT), conciliação de medicamentos ou revisão da farmacoterapia e outros, se formam pelo conhecimento desse profissional em reconhecer, precaver e definir adversidades relativas aos medicamentos (Rossignoli et al., 2019). O farmacêutico ainda partilha com os demais profissionais da saúde outros serviços, como é o caso da educação em saúde e do rastreamento. As técnicas de suporte, também podem ser providos, como a investigação e/ou monitoramento de fatores clínicos, a execução de pequenos curativos, a ordenação dos medicamentos em uso pelo paciente etc. (França et al., 2021).

No cuidado contínuo ao paciente, família e comunidade, a ação do farmacêutico é direcionada pela área de conhecimento chamada Farmácia Clínica. Assim, compete salientar a Lei n ${ }^{\circ}$ 13.021/2014, que admite a farmácia como uma unidade de fornecimento de serviço à saúde, enumerando várias incumbências do farmacêutico no método de cuidado. Destaca-se ainda, a Resolução do Conselho Federal de Farmácia (CFF) no 585/2013, que determina os benefícios clínicos, ou seja, as garantias e responsabilidades do profissional quanto ao seu desempenho clínico voltado ao paciente (CFF, 2013; Gadelha et al., 2020). 
Neste sentido, o estudo teve como intuito elaborar uma revisão integrativa acerca dos principais SF atribuídos ao paciente, família e comunidade no Brasil.

\section{Metodologia}

A coleta de informações foi processada com a investigação bibliográfica e consulta em banco de dados: Biblioteca Virtual em Saúde (BVS), Literatura Latino-Americana e do Caribe em Ciências da Saúde (LILACS) e Scientific Eletronic Library Online (Scielo) para alcance de saberes teóricos sobre o tema a ser desenvolvido na pesquisa (Mendes et al., 2019). Usou-se como indicador os termos: serviços farmacêuticos, farmácia clínica e farmacêutico.

Os fatores de inserção determinados para seleção dos artigos foram: estudos que publicaram textos integrais e disponíveis nos idiomas português e inglês, com livre acesso e conduzido nos últimos onze anos (2010 a 2021).

Para a organização da amostra, foi feita a análise dos artigos conforme os critérios de inclusão, seguida por escolha baseada na literatura dos títulos e resumos, com posterior exclusão dos estudos que não se enquadrassem na temática da revisão ou que estivessem duplicados nas bases de dados.

\section{Resultados e Discussão}

O fornecimento e a continuação de serviços e produtos para a prevenção das pessoas formam um problema de saúde pública (Mieiro et al., 2019). A proporção da demanda de acesso e uso de meios terapêuticos e propedêuticos é constantemente superior a capacidade de financiamento e abastecimento dos sistemas de saúde. O episódio de transformação demográfica pertinentes ao envelhecimento populacional, o aumento do predomínio de condições crônicas e, por consequência, o acréscimo do uso incessante de medicamentos, estabelecem as fundamentais razões ligados à crise nos sistemas de saúde (Barbareto et al., 2019).

Neste caso, modificar o formato de favorecer o cuidado à saúde das pessoas e fomentar benefícios potenciais de cada profissional, instigam a sustentabilidade desses sistemas. Em direção contrária à demanda do sistema de saúde, vê-se a presença de um espaço entre o benefício potencial dos medicamentos e o seu concreto valor terapêutico. O farmacêutico, apesar de simbolizar um profissional habilidoso para o sistema de saúde, é subutilizado (Carvalho et al., 2017).

O exercício do farmacêutico no cuidado direto ao paciente, família e comunidade, com o intuito de conter a morbimortalidade relativa ao uso dos medicamentos, propiciando a saúde e prevenindo a doença, é um desafio para os sistemas de saúde brasileiro. Diversos países como Canadá, Reino-Unido, Austrália, Nova Zelândia, Espanha, Portugal, Holanda, Suíça, entre outros, instigaram o aumento da atividade clínica do profissional como técnica para conquista dos melhores resultados para o paciente (Cazarim et al., 2016; Mourão et al., 2019).

Neste cenário, há desafios para alargar a atuação do farmacêutico no sistema de saúde como, por exemplo, a concordância de ideias pertinentes às "seleções de SF". Isto contribuirá para a solidificação de diretrizes de atuação ocupacional e para regulação de métodos de trabalho, propiciando o crescimento dos resultados e da formação trabalhista (Guimarães et al., 2021).

Inúmeras categorias de SF, concebidas no contexto da atenção à saúde, encontram-se expostos e apreciados positivamente no desenvolvimento da qualidade do processo do uso de medicamentos e nas consequências terapêuticos para o usuário. Chisholm-Burns et al. (2010), em uma revisão sistemática com meta-análise, que abrangeu 224 estudos, examinaram a contribuição do farmacêutico nos sistemas de saúde. Como resultado, acharam indicativos de excelentes desfechos clínicos no manejo dos problemas de saúde e episódios pertencentes à segurança da farmacoterapia, tais como: diminuição dos valores de hemoglobina glicosilada, redução nos níveis de colesterol, atenuação da demanda por serviços de emergência, redução da taxa 
de hospitalização, do tempo de estadia hospitalar, dos eventos adversos a medicamentos, do índice de massa corporal e da mortalidade.

De modo igual, existem indicativos de obtenção de efeitos positivos no AFT. Desde 1998, 15.000 pacientes de um plano de saúde americano (Fairview Health Services), exibiram melhora significativa dos padrões clínicos, revelando uma economia para o plano de saúde, onde, para cada dólar aplicado neste serviço, o sistema receberia entre 2,5 e 12 dólares de retorno (Freitas et al., 2021).

Há também, vários estudos sobre as consequências positivas do desempenho do farmacêutico voltada a pessoas com asma (Rezende et al., 2020). Estes afirmam que o profissional pode cooperar para o acréscimo de entendimento, promoção de competências na manipulação dos dispositivos inalatórios, melhoria nos efeitos clínicos e na qualidade de vida dessas pessoas (Hohl et al., 2017). Da mesma forma, encontram-se sinais de que este profissional quando incorporado à atenção primária à saúde (APS) pode oferecer o serviço de revisão da farmacoterapia com avanço nos desfechos positivos de saúde para os pacientes. Estudos de revisão sistemática sugerem que serviços providos por farmacêuticos, como a revisão da farmacoterapia, simplificação de regime terapêutico e auxílio para a administração de medicamentos, são essenciais para conter a prescrição imprópria e evitar a subutilização de medicamentos ou uso de fármacos eventualmente perigosos, informando interações medicamentosas que promovam a insegurança aos pacientes (Galato et al., 2021).

A conciliação de medicamentos tem exibido enorme impacto na prevenção de eventos adversos relativos aos medicamentos, limitando as discrepâncias não intencionais em alterações de níveis de atenção, serviços ou profissionais da saúde, possibilitando assim, a contenção desse tipo de falha de medicação em cerca de 70\%. Kitts et al. (2014), em estudo de revisão sistemática, dizem que os farmacêuticos, é parte complementar da equipe multidisciplinar, possuindo uma posição de destaque na educação dos pacientes.

Outro SF é o manejo de problemas de saúde autolimitados. Em uma revisão sistemática, identificou-se a resolução integral dos problemas entre $68 \%$ e $94 \%$ das situações tratadas pelo farmacêutico, sendo que, o gasto médio da consulta diferiu de 2,26 a 25,28 dólares. Houve ainda, a contenção de 7,9\% na busca pelos serviços de urgência ou emergência para o auxílio de problemas de saúde autolimitados no grupo tratado pelo farmacêutico (Anderson et al., 2019).

No contexto atual, observa-se que o subdiagnóstico de muitas doenças, principalmente as crônicas, é ainda visto como alto, ou seja, infinitas chances de cuidado são desperdiçadas frequentemente (Botelho et al., 2017). Estudos confirmam que os farmacêuticos, ocupam lugar tático no sistema, para distinguir pacientes sob risco e encaminhá-lo primeiramente o médico para a execução de diagnóstico prévio, por intermédio do fornecimento do serviço de rastreamento em saúde (Dias et al., 2019). Assim, diante os resultados exibidos, tona-se urgente alargar a oferta dos SF em nosso país.

\section{Considerações Finais}

Este estudo, mostra desfechos clínicos positivos feitos por farmacêuticos através dos seus serviços. A introdução dos SF expõe um maior reconhecimento das competências do profissional no sistema de atenção à saúde, e o enaltecimento do impacto de sua atuação na melhora da farmacoterapia, no aprimoramento dos resultados em saúde, na prevenção de doenças e na ascensão de saúde da população brasileira.

Para uma maior compressão, aconselha-se que seja assinalado nos próximos estudos, os principais problemas enfrentados pelo profissional na inserção dos SF em organizações publicas e privadas no Brasil e, recomendações de melhoria para o processo de trabalho, aptidões profissionais para o fornecimento de cada serviço nas instituições de saúde do Brasil. 


\section{Referências}

Anderson SL, Marrs JC, Vande GJP, Hanratty R. (2019). Implementation of a Clinical Pharmacy Specialist-Managed Telephonic Hospital Discharge Follow-Up Program in a Patient-Centered Medical Home. Population Health Management, 16 (4): 235-241.

Araújo PS, Costa EA, Junior AAG, Acurcio FA, Guibu IA, Álvares J, Costa KS, Karnikowski MGO, Soeiro OM, Leite SN. (2017). Atividades farmacêuticas de natureza clínica na atenção básica no Brasil. Revista de Saúde Pública, 51 Supl 2:6s.

Barberato LC, Scherer MDDA, Lacourt RMC. (2019). The pharmacist in the Brazilian Primary Health Care: insertion under construction. Ciências \& Saúde Coletiva, 24 (10): 3717-3726.

Botelho JA, Roese FM. (2017). Intervenções Realizadas Pelo Farmacêutico Em Uma Unidade De Pronto Atendimento Médico. Revista Brasileira de Farmácia Hospitalar e Serviços de Saúde, 8 (1): 34-36.

Carvalho MN, Álvarez J, Costa KS, Junior AAG, Acurcio FA, Costa EA, Guibu IA, Soeiro OM, Karnikowski MGO, Leite SN. (2017). Força de trabalho na assistência farmacêutica da atenção básica do SUS, Brasil. Revista de Saúde Pública, 51 Supl 2: 16s.

Cazarim MS, Freitas O, Penaforte TR, Achcar A, Pereira LRL. (2016). Impact Assessment of Pharmaceutical Care int the Management of Hypertension and Coronary Risk Factors after Discharge. PLoS ONE, 11 (6): e0155204.

Chisholm-Burns MA, Zivin JSG, Lee JK, Spivey CA, Slack M, Herrier RN, Lipsy EH, Abraham I, Palmer J. (2010). Economic effects of pharmacysts on health outcomes in the United States: systematic review. American Journal of Health-System Pharmacy, 67 (19): 1624-34.

Conselho Federal de Farmácia. Resolução n 585 de 29 de agosto de 2013: Regulates clinical assignments pharmacist and makes other arrangements.

Dias D, Wiese LPL, Pereira EM, Fernandes FM. (2019). Avaliação de intervenções clínicas farmacêuticas em uma UTI de um Hospital Público de Santa Catarina. Revista Brasileira de Farmácia Hospitalar e Serviços de Saúde, 9 (3): 1-5.

Fernandes LL (2019). A importância do farmacêutico hospitalar juntamente com a equipe multidisciplinar na Unidade de Terapia Intensiva (UTI). Revista Farol, 8 (8): 5-21.

Maioli NA, Santos HCB. (2018). Intervenções Farmacêuticas E Sua Importância Na Segurança Do Paciente Hospitalizado. Colloquium Vitae, 10 (2): $35-40$.

França C, Andrade LG. (2021). Performance of the Pharmacist in health care in community Pharmacies. Revista Ibero-Americana de Humanidades, Ciência e Educação, 7 (9): 10-20.

Freitas GR, Santos JU, França JS, Pinto RS, Seixas BV. (2021). Economic impact of pharmacysts' interventions in asthma management: a systemmatic review. Revista Brasileira de Farmácia Hospitalar e Serviços de Saúde, 21 (3): 0587.

Gadelha CAG, Braga PSC, Montenegro KBM, Cesário BB. (2020). Access to vaccines in Brazil and the global dynamics of the Health Economic-Industrial Complex. Reports in Public Health; 36 Sup 2: e00154519.

Galato D., Alano G. M., Trauthman S. C., Vieira A. C. (2021). A dispensação de medicamentos: uma reflexão sobre o processo para prevenção, identificação e resolução de problemas relacionados à farmacoterapia. Revista Brasileira de Ciências Farmacêuticas, 44 (3): 629-640.

Guimarães PHD, Pacheco RP, Morais, YJ. (2021). Pharmaceutical care and the use of Over-the-Counter Medications (MIPs). Research, Society and Development, 10 (12): e485101220405.

Hohl CM, Partovi N, Ghement I. (2017). Impact of early in-hospital medication review by clinical pharmacists on health services utilization. PlosOne, 12 (2): e0170495.

Kitts NK, Reeve AR, Tsul L. (2014). Care transitions in elderly heart failure patients: current practices and the pharmacyst's role. Consultant Pharmacist, 29 (3): $179-90$.

Melo AC, Trindade GM, Freitas AR, Resende KA, Palhano TJ. (2021). Community pharmacies and pharmacists in Brazil: A missed opportunity. Pharmacy Practice. 19 (2): 2467.

Mendes KDS, Silveira RCCP, Galvão CM. (2019). Uso de gerenciador de referências bibliográficas na seleção dos estudos primários em revisão interativa. Revista Texto e Contexto Enfermagem, 28 (1): e20170204.

Mieiro DB, Oliveira ÉBC, Fonseca REP, Mininel VA, Zem-Mascarenhas SH, Machado RC. (2019). Strategies to minimize medication errors in emergency units: an integrative review. Revista Brasileira de Enfermagem, 72 (suppl 1): 307-314.

Mourão KQ, Oliveira AMM. (2019). Notificação de eventos: avanços e desafios no contexto da segurança do paciente. Revista Eletrônica Acervo Saúde, 24: 1-6.

Rezende LHO, Gehrke FDS, Silva MA, Carneiro AMF, Abreu RM, Monteiro CN, Takei K. (2020). Prescrição de medicamentos: uma análise para a implantação da prescrição eletrônica ambulatorial. Revista Eletrônica Acervo Saúde, 12 (9): e3638.

Rossignoli P, Pontarolli D, Corrêa LG, Germano LJ, Pontarolo R, Fernandez-Llimos F. (2019). Innovating in clinical pharmacy services at the specialized component of pharmaceutical management in the state of Paraná. Revista de Saúde Pública do Paraná, 2 (1): 125-139.

Souza LB, Souza DM, Souza SM, Silva DR, Aguilar NC. (2018). Importância do farmacêutico clínico no uso seguro e racional de medicamentos no âmbito hospitalar. Revista Pensar Acadêmico, 16 (1): 109-124. 